\title{
Dry powder inhalers: upcoming platform technologies for formulation development
}

\author{
Piyush Pradeep Mehta*,1 (iD \\ ${ }^{1}$ Department of Quality Assurance, Poona College of Pharmacy, Bharati Vidyapeeth University, Paud Road, Rambaug Colony, \\ Erandwane, Pune 411038, Maharashtra, India \\ *Author for correspondence: piyu053@gmail.com
}

"The present article is focused on the cutting-edge platform technologies for effective therapeutic delivery that can counter the difficulties during treatment of pulmonary ailments."

First draft submitted: 21 August 2019; Accepted for publication: 24 September 2019; Published online: 14 October 2019 Keywords: dry powder inhaler $\bullet$ hot melt extrusion $\bullet$ inhaled therapeutics $\bullet$ lactose $\bullet$ particle engineering $\bullet$ perflu-
oropolyethers $\bullet$ platform technologies $\bullet$ pulmonary delivery $\bullet$ PulmoSphere ${ }^{\top M} \bullet$ Technosphere ${ }^{\circledR}$

Pulmonary diseases pose a major challenge in existing healthcare [1]. In spite of various treatment strategies, it remains the most significant cause of death across the globe [2,3]. Although pulmonary diseases appear chronic with moderately slow progress, the span of treatment and the rising of treatment burden impede the quality of patient life. Eventually, pulmonary diseases will result in a serious socioeconomic burden [1-3].

Growing advances in material sciences, nanotechnology, molecular biology and similar scientific domains provide us a platform to treat various intrapulmonary and extrapulmonary diseases such as asthma, chronic obstructive pulmonary disease (COPD), tuberculosis, cystic fibrosis, hypertension and diabetes with better therapeutic outcomes [4-8]. The present article is focused on the cutting-edge platform technologies for effective therapeutic delivery that can counter the difficulties during treatment of pulmonary ailments.

\section{Need of platform technologies}

Dry powder inhalers (DPIs) are the unique combination of a micronized drug $(0.5-5 \mu \mathrm{m})$; carrier molecule (80$150 \mu \mathrm{m})$ and inhaler device. In DPIs, the carrier molecule plays a crucial part during handling, dispensing and actuation due to small mass of the drug $(18-500 \mu \mathrm{g})$ within the finished product. DPI manufacturing involves loose adherence of the drug particles to the carrier molecules, then in inhalation maneuver, drug particles should easily detach from the carrier surface and be available for deposition within the pulmonary airways. Such satisfactory drug-delivery performance is defined by the fundamental cohesive-adhesive balance within the drug and carrier molecule. Moreover, DPIs therapeutic performance is also governed by carrier morphology, device configuration and disease progression [9,10]. Hence, various active formulators and clinicians have investigated different novel technologies to improve the overall performance of DPIs.

\section{Platform technologies}

Platform technologies are novel drug delivery systems, that are engineered with the objective to surpass the drawbacks of conventional carrier-based DPIs and to achieve superior pharmacological outcomes. For instance, Novartis's (Basel, Switzerland) PulmoSphere ${ }^{T M}$ or MannKind's (CA, USA) Technosphere ${ }^{\circledR}$ systems have made a remarkable impact on the treatment of pulmonary diseases, which benefits pharmaceutical industries. The platform technologies studied and analyzed for DPIs are summarized below.

\section{Particle replication in nonwetting templates}

Particle replication in nonwetting templates (PRINT) is a unique, roll-to-roll, high-resolution, soft lithographybased molding technology that permits the design and engineering of defined microparticles as well as nanoparticles. It makes use of low surface energy novel compounds, such as fluoropolymers and photocurable perfluoropolyethers. 
PRINT is a plug-and-play approach that molds particles to tailored physicochemical properties [11]. Garcia et al. engineered zanamivir microparticles using PRINT technology, which demonstrated a 3.19-fold improvement in fine particle fraction (FPF) compared with conventional DPI [12]. Moreover, an in vivo lung deposition study demonstrated that PRINT aerosols (lactose:albumin:leucine; 64:32:4) tagged with technetium-99 displayed uniform lung deposition with good control over mass median aerodynamic diameter $(1.5 \mu \mathrm{m})$ [12]. Last, the unique features of PRINT may facilitate a remarkable transformation in particle morphology for improved pulmonary drug delivery.

\section{Inkjet-printed aerogel particles}

Inkjet printing (IJP) is a novel material processing technology that structures a digital image by impelling droplets of ink onto suitable substrates. IJP offers precise control over the droplet size, particle size and morphology. IJP in the 'drop-on-demand' style allows a wide range of applications ranging from engineering of microparticles to cell deposition for regenerative medicine [13]. López-Iglesias et al. fabricated thermal IJP-based salbutamol sulfate-loaded alginate aerogel microspheres (Salbutamol sulfate [SS]-aerogel) and demonstrated that highly porous and lightweight nanoporous particles $(2.4 \mu \mathrm{m})$ exhibited an improved FPF of $49.70 \%$ and sustained a drug-release pattern $(>10 \mathrm{~h})$ in phosphate buffer saline (pH 7.4), owing to the unique structural features of aerogel [14]. IJP-based microparticles can be further utilized to design personalized aerosols with improved control over FPF and mass median aerodynamic diameter.

\section{PulmoSphere ${ }^{T M}$ platform}

PulmoSphere is the clinically proven, proprietary formulation technology of Novartis (Basel, Switzerland). It contains small porous particles with a typical sponge structure, which is fabricated by spray drying. The key ingredients of PulmoSphere are endogenous distearoylphosphatidylcholine and calcium chloride (2:1). The hydrophobic facade, which is materialized by lipids, assists in reducing interparticle cohesive forces, which can provide excellent powder dispersibility and improved aerosols performance. PulmoSphere technology involves aerodynamically suitable particles that are obtained in three ways: solution-based, suspension-based and carrier-based formulations. To date a variety of molecules ( $>60$ ) has been successfully incorporated into PulmoSphere to achieve a superior dose consistency, systemic targeting, deep lung deposition and fabrication of fixed dose combinations [15]. Briefly, PulmoSphere represented a significant platform for nurturing product opportunities in the pulmonary domain.

\section{Technosphere ${ }^{\circledR}$ technology}

MannKind's Technosphere technology is a multipurpose pulmonary delivery platform cultivated from substituted cyclized dipeptides, for example, fumaryl diketopiperazine (FDKP). Acid-induced, intermolecular, self-assembly of FDKP is materialized for a particle matrix (microcrystalline plates structure) of Technosphere. It is a 3D sphere with particle size $(2-5 \mu \mathrm{m})$ that is suitable for pulmonary delivery. After inhalation, Technosphere dissolves quickly and releases an active payload into the lungs, owing to FDKP's high solubility at $\mathrm{pH} \geq 6$. Numerous Technosphere formulations are under clinical trials for diabetes mellitus (Types 1 and 2), chronic obstructive pulmonary disease, asthma and pulmonary infections [16]. The unique, single, excipient-based Technosphere technology has the potential to improve the aerosolization performance of inhaled therapeutics.

\section{Inhaled small particles easily respirable \& emittable}

Inhaled small particles easily respirable and emittable (iSPERSE ${ }^{T M}$ ) technology involves the use of pharmaceutically acceptable inorganic and organic salt-based excipients for pulmonary delivery. iSPERSE technology involves, aqueous or organic dispersion of salt-based excipients (magnesium stearate, leucine) and different drugs such as tobramycin and salmeterol xinafoate that are spray dried to obtain geometrically small, dense and aerodynamically a stone particle technology particles. iSPERSE-derived particles exhibit flow rate independent capsule emptying and drug emission. The addition of salt-based excipients aid in the regulation of the cohesive-adhesive balance and enhance the overall dispersibility of particles, irrespective of their morphology. iSPERSE technology allows the delivery of large masses of drugs (0.6-95\%) through a passive DPI that utilizes a one-step spray drying process. Hence, iSPERSE has a better potential to deliver a wide range of molecules during pulmonary therapy [17]. 


\section{Hot melt extrusion}

Hot melt extrusion (HME) is receiving huge attention in the healthcare sector owing to its proven capability for efficient multifunctional products. The versatile nature of HME aids the engineering of products ranging from powders to medical devices [18]. Recently, Lin et al. explored an HME-based solvent-free fine solid crystal suspension technique for the development of itraconazole inhalation powder. Initially, jet-milled itraconazole was mixed with mannitol (20:80) and subjected to a conical co-rotating, twin-screw extruder to generate extrudates. These extrudates were jet-milled to achieve a aerodynamically suitable particle size $(2.19 \mu \mathrm{m})$. DPIs utilized an Turbospin ${ }^{\circledR}$ (PH\&T, Milan, Italy) inhaler $(60 \mathrm{l} / \mathrm{min})$ and demonstrated a superior FPF of 50.69\% with stable aerodynamic performance at accelerated storage conditions for 6 months. Enhancement in aerosolization performance of irregular-shaped nonspherical fine solid crystal suspension (FSCS)-based particles, may be attributed to a decrease in the particle's agglomeration [19]. In brief, HME presented an attractive formulation approach to augment the aerodynamic performance and stability of a drug molecule.

Briefly, platform technologies are versatile to defeat the limitations of conventional DPIs. The tailor-made approach of platform technologies facilitates the fabrication of multifunctional inhaled dosage forms, for a wide range of active therapeutics. Ultimately, the exclusive features of these platform technologies may facilitate a transformation in the design and delivery of DPIs. However, there is essential to methodically validate these platform technologies for improved pulmonary functioning. The focus of upcoming investigations will relies upon superior animal models for improved in vivo understanding; simulation approaches to grasp device/formulation interface; new regulatory requirements; and chemistry, manufacturing and control challenges. In conclusion, adequate strength from working scientists, their insights and the diversity of scientific domain, needs to be cultured to continue the fight against pulmonary diseases.

\section{Acknowledgments}

The author is thankful to Bharati Vidyapeeth University, Poona College of Pharmacy, Pune-38, India for support and institutional facilities.

\section{Financial \& competing interests disclosure}

The author has no relevant affiliations or financial involvement with any organization or entity with a financial interest in or financial conflict with the subject matter or materials discussed in the manuscript. This includes employment, consultancies, honoraria, stock ownership or options, expert testimony, grants or patents received or pending, or royalties.

No writing assistance was utilized in the production of this manuscript.

\section{References}

1. Mehta P, Kadam S, Pawar A, Bothiraja C. Dendrimers for pulmonary delivery: current perspectives and future challenges. New J. Chem. 43(22), 8396-8409 (2019).

2. Mehta P, Bothiraja C, Kadam S, Pawar A. Potential of dry powder inhalers for tuberculosis therapy: facts, fidelity and future. Artif. Cells Nanomed. Biotechnol. 46(3), 791-806 (2018).

3. Mehta P, Bothiraja C, Mahadik K, Kadam S, Pawar A. Phytoconstituent based dry powder inhalers as biomedicine for the management of pulmonary diseases. Biomed. Pharmacother. 108, 828-837 (2018).

4. Mehta P. Multi-dose dry powder inhaler: advance technology for drug delivery to airways. Indian Drugs(2018). https://www.indiandrugsonline.org/accepted-article-details?id=MTE0MDM

5. Mehta P. Dry powder inhalers: a focus on advancements in novel drug delivery systems. J. Drug Deliv. 2016, 1-17 (2016).

6. Keil TW, Merkel OM. Dry powder inhalation of siRNA. Ther. Deliv. 10(5), 265-267 (2019).

7. Wylie JL, House A, Mauser PJ et al. Inhaled formulation and device selection: bridging the gap between preclinical species and first-in-human studies. Ther. Deliv. 9(5), 387-404 (2018).

8. Mehta P. Imagine the superiority of dry powder inhalers from carrier engineering. J. Drug Deliv. 1-19 (2018).

9. Mehta P, Bothiraja C, Kadam S, Pawar A. Effect of USP induction ports, glass sampling apparatus, and inhaler device resistance on aerodynamic patterns of fluticasone propionate-loaded engineered mannitol microparticles. AAPS PharmSciTech 20(5), 197 (2019).

10. Newman SP. Drug delivery to the lungs: challenges and opportunities. Ther. Deliv. 8(8), 647-661 (2017).

11. Xu J, Wong DH, Byrne JD, Chen K, Bowerman C, DeSimone JM. Future of the particle replication in non wetting templates (PRINT) technology. Angew. Chem. Int. Ed. Engl. 52(26), 6580-6589 (2013).

12. Garcia A, Mack P, Williams $S$ et al. Microfabricated engineered particle systems for respiratory drug delivery and other pharmaceutical applications. J. Drug Deliv. 2012, 941243 (2012). 
13. Scoutaris N, Ross S, Douroumis D. Current trends on medical and pharmaceutical applications of inkjet printing technology. Pharm. Res. 33(8), 1799-816 (2016).

14. López-Iglesias C, Casielles AM, Altay A, Bettini R, Alvarez-Lorenzo C, García-González CA. From the printer to the lungs: Inkjet-printed aerogel particles for pulmonary delivery. Chem. Eng. J. 357, 559-566 (2018).

15. Weers J, Tarara T. The PulmoSphere ${ }^{\top M}$ platform for pulmonary drug delivery. Ther. Deliv. 5(3), 277-295 (2014).

16. Sarala N, Bengalorkar G, Bhuvana K. Technosphere: new drug delivery system for inhaled insulin. Future Prescriber 13(1), 14-16 (2012).

17. Sung JC, Manzanedo D, Ku AT et al. iSPERSE ${ }^{\mathrm{TM}}$ : formulation and in vitro characterization of a novel dry powder drug delivery technology. Resp. Drug Deliv. Eur. 411-414 (2011).

18. Wilson M, Williams MA, Jones DS, Andrews GP. Hot-melt extrusion technology and pharmaceutical application. Ther. Deliv. 3(6), 787-797 (2012).

19. Lin L, Quan G, Peng T et al. Development of fine solid-crystal suspension with enhanced solubility, stability, and aerosolization performance for dry powder inhalation. Int. J. Pharm. 533(1), 84-92 (2017). 